\title{
The Multi-state Model Fusion Algorithm for GNSS Navigation
}

\author{
Dawen Zhang \\ Dept. IC design \\ Shanghai Fudan-Holding Hualong Microsystem Technology \\ Shanghai, china \\ e-mail: zhangdawen@fkhl.sh.cn
}

\begin{abstract}
A novel fusion algorithm, termed as the multi-state model fusion filter (MSMF), was established for the global navigation satellite systems (GNSS) navigation. Firstly, a new state space model was presented by adopting the polynomial predictive idea and state dimension expansion. It was established without the knowledge of the original state dynamics, that is, no matter the original state propagation was known or not. Then two local estimation results were obtained based on the original model and the proposed model. Whereafter, the local results were fused to get the global estimation. The simulation results of the global positioning system (GPS) navigation verified the effectiveness of the proposed method.
\end{abstract}

Keywords-GNSS, Kalman filter, data fusion, polynomial predictive, state space model

\section{INTRODUCTION}

The global navigation satellite systems (GNSS) may provide user's position and velocity from the delay and Doppler of direct-sequence signals sent by satellites [1]. In the last decade, the applications that utilize GNSS have expanded amazingly, especially the use of global positioning system (GPS), one of the famous GNSS, because of its usefulness and low cost. Recently a number of filtering algorithms have been presented and used for GNSS navigation. Usually they offer efficient and iterative means of combining information from sensor(s) to estimate the state such as the position, velocity and acceleration of the user. Among the algorithms, a process model is commonly adopted to predict the future state. Then the estimate of the state is updated by using the measurements. For example, the Kalman filter (KF) was used for the purpose of improvement of the GPS positioning precision under small number of satellites used for calculating in [2]. The authors adapted the KF to GPS positioning calculating when they could see only 3 satellites in the sky. It is concluded that the positioning precision of the proposed method was improved as compared with the algorithm of least mean square. In [3], the particle filter (PF) was applied to the relative position estimation using GPS carrier-phase measurements. The probability density function of the position was estimate by sampling from the position space with the particle filter. In [4], the KF was used for GPS/magnetometer integrated navigation system, which improved the accuracy. In [5], the KF was adopted for GPS measurement of a motorcycle trajectory, where the trajectory with excellent continuity and stability were obtained even in the situation that the observation data were missed within a few seconds. In addition, the EKF was used to make the trajectory smooth and the trajectory with high accuracy. Also the extended Kalman filter (EKF) was used in a GNSS, gyro and odometer loosely coupled data fusion architecture for a road vehicle [6].

Note that the existed KF, EKF, PF work well only if the state transition density is known well. Otherwise, the filters may be divergent, i.e., they could not provide the user's correct position or velocity in GNSS navigation. However, the user's path may be uncertain in practice. Furthermore the existed filtering algorithms use only one process model so that there have been no estimation fusion in GNSS navigation.

In this paper, the multi-state model fusion filter (MSMF) is proposed to solve the above problems. We firstly adopt the idea of the polynomial predictive filtering to construct a new process model for GNSS navigation by expanding the dimension of the state, i.e., the expanded state, which consists the original state at the current time step and those of several backward time steps. In this way, we may obtain the expanded state dynamics for GNSS navigation whether the original state transition density is known or not. Then two local estimation results were obtained based on the original model and the proposed model. Whereafter, the global estimation is obtained by fusing the two local results. Simulation results show that when the true state dynamics are not known well, the proposed method still works well while the existed central difference filter does not.

\section{The Existed Polynomial PREdictive FILTER}

According to Weierstrass approximation theorem, for a continuous real-valued signal, it can always be divided into lots of sequential closed and bounded intervals in which the signal can be uniformly approximated by low degree polynomials to any degree of accuracy. Suppose that the signal $x_{k}$ can be modeled as a polynomial with low degree $L$, then we have [7]

$$
x_{k}=\sum_{l=0}^{L} p_{l} k^{l},
$$

where $p_{l}$ is the polynomial coefficients. We may have

$$
x_{k}=\sum_{l=0}^{L} p_{l} k^{l}+w_{k}
$$


when signal $x_{k}$ does not fit the polynomial model exactly and $w_{k}$ is an error term.

It turns out that predictions using polynomial models can be done by taking a weighted average of the past few values of the signal and that the weighted coefficients are not dependent on the signal, but only on the values assigned to polynomial degree, number of prediction steps and the length of the filter [7, 8], i.e., for exactly polynomial signals, a future value $x_{k+N}$ can be obtained as

$$
X_{k+N}=\sum_{m=0}^{M-1} h_{m} x_{k-m},
$$

where, $N$ and $M$ denote the number of prediction steps and the length of the filter respectively, $h_{m}$ are the filter coefficients. For example, with $N=1, L=1, h_{m}$ becomes[7, 8]

$$
h_{m}=\frac{4 M-6 m-4}{M(M-1)} \text {. }
$$

\section{THE NEW MODEL}

\section{A. The process model}

Suppose that the true model is

$$
x_{k+1}=f_{k}\left(x_{k}\right)+n_{k} \text {, }
$$

where, $n_{k}$ is the process noise and $f_{k}($.$) is unknown. By$ the knowledge of polynomial predictive filter reviewed in last section, we rewrite (5) as

$$
X_{k+1}=\sum_{m=0}^{M-1} h_{m} x_{k-m}+v_{k+1},
$$

where, $v_{k+1}$ is a noise term and it is zero if $x_{k}$ is a strictly polynomial signal. Further more, (6) can be rewritten as an expanded form

$$
\bar{X}_{k+1}=F_{p p f} \bar{X}_{k}+\bar{V}_{k+1} \text {, }
$$

where,

$$
\begin{gathered}
\bar{X}_{k}=\left[x_{k}, x_{k-1}, \cdots, x_{k-M+1}\right]^{T}, \\
F_{p p f}=\left[\begin{array}{cccc}
h_{0} & h_{1} & \cdots & h_{M-1} \\
1 & 0 & \cdots & 0 \\
\cdots & \cdots & \cdots & \cdots \\
0 & \cdots & 1 & 0
\end{array}\right], \\
\bar{V}_{k}=\left[v_{k}, v_{k-1}, \cdots, v_{k-M+1}\right]^{T} .
\end{gathered}
$$

Suppose that $v_{k}$ is white Gaussian with mean zero and covariance $Q$, and it is independent at different time steps. Then $\bar{V}_{k}$ is also white Gaussian with mean zero and covariance $Q_{p p f}$, i.e.,

$$
Q_{p p f}=\operatorname{blkdiag}(Q, \cdots, Q)_{M l \times M l},
$$

where, $l$ is the dimension of the original state.

\section{B. The measurement model}

Suppose that the original pseudorange measurement model is as follows [9]

$$
Y_{k}=H\left(x_{k}\right)+W_{k} \text {, }
$$

$$
H\left(x_{k}\right)=\left[\begin{array}{c}
\sqrt{\left(x_{1 k}-x_{k}\right)^{2}+\left(y_{1 k}-y_{k}\right)^{2}+\left(z_{1 k}-z_{k}\right)^{2}} \\
\sqrt{\left(x_{2 k}-x_{k}\right)^{2}+\left(y_{2 k}-y_{k}\right)^{2}+\left(z_{2 k}-z_{k}\right)^{2}} \\
\sqrt{\left(x_{3 k}-x_{k}\right)^{2}+\left(y_{3 k}-y_{k}\right)^{2}+\left(z_{3 k}-z_{k}\right)^{2}} \\
\sqrt{\left(x_{4 k}-x_{k}\right)^{2}+\left(y_{4 k}-y_{k}\right)^{2}+\left(z_{4 k}-z_{k}\right)^{2}}
\end{array}\right]+c t_{k},
$$

where, $x_{k}, y_{k}, z_{k}$ are the $x, y, z$ positions of the user, $x_{i k}, y_{i k}, z_{i k}$ are the $x, y, z$ positions of $i$ th satellites, $i=1,2,3,4, c$ is the light speed, $t_{k}$ is the clock bias, $W_{k}$ is white Gaussian with mean zero and covariance $R$. Then we may rewrite (12) in an expanded form corresponding to (8), i.e.,

$$
\bar{Y}_{k}=\bar{H}\left(\bar{X}_{k}\right)+\bar{W}_{k},
$$

where,

$$
\begin{aligned}
& \bar{H}\left(\bar{X}_{k}\right)=\left[\begin{array}{llll}
H\left(x_{k}\right) & H\left(x_{k-1}\right) & \cdots & H\left(x_{k-M+1}\right)
\end{array}\right]^{T} . \\
& \bar{W}_{k}=\left[\begin{array}{lll}
W_{k}, W_{k-1}, \cdots, W_{k-M+1} & ]^{T}
\end{array}\right.
\end{aligned}
$$

Suppose that $W_{k}$ is independent at different time steps, the covariance of $\bar{W}_{k}$ can be expressed as

$$
\bar{R}_{k}=\operatorname{blkdiag}(R, \cdots, R)_{4 M \times 4 M} .
$$

IV. The Proposed Multi-state Model Fusion Filter (MSMF)

A. The existed model

Suppose that the existed model is as follows [10],

$$
x_{k}=F_{k} x_{k-1}+\Gamma_{k} v_{k},
$$

where, $x_{k}=\left[\begin{array}{llllll}p x_{k} & p y_{k} & p z_{k} & v x_{k} & v y_{k} & v z_{k}\end{array}\right], \quad p x_{k}$, $p y_{k}$ and $p y_{k}$ are the user position in $x, y, z$ axes respectively, $v x_{k}, v y_{k}$ and $v y_{k}$ are the corresponding speed, and

$$
\begin{aligned}
F_{k} & =\left[\begin{array}{cccccc}
1 & 0 & 0 & d T & 0 & 0 \\
0 & 1 & 0 & 0 & d T & 0 \\
0 & 0 & 1 & 0 & 0 & d T \\
0 & 0 & 0 & 1 & 0 & 0 \\
0 & 0 & 0 & 0 & 1 & 0 \\
0 & 0 & 0 & 0 & 0 & 1
\end{array}\right], \\
\Gamma_{k} & =\left[\begin{array}{cccc}
d T^{2} / 2 & 0 & 0 \\
0 & d T^{2} / 2 & 0 \\
0 & 0 & d T^{2} / 2 \\
T & 0 & 0 \\
0 & T & 0 \\
0 & 0 & T
\end{array}\right],
\end{aligned}
$$

$d T$ is the time step interval. 


\section{B. The MSMF}

By applying the extended Kalman filter (EKF) to the proposed equation (7) and (14), we have

$$
\begin{aligned}
& \hat{\bar{X}}_{k+1 \mid k}=F_{p p f} \hat{\bar{X}}_{k \mid k} \\
& P_{k+1 \mid k}=F_{p p f} P_{k \mid k} F_{p p f}^{T}+Q_{p p f} \\
& K_{k+1}=P_{k+1 \mid k} \bar{H}_{p p f}^{T}\left(\bar{H}_{p p f} P_{k+1 \mid k} \bar{H}_{p p f}^{T}+\bar{R}_{k+1}\right)^{-1}, \\
& \hat{\bar{X}}_{k+1 \mid k+1}=\hat{\bar{X}}_{k+1 \mid k}+K_{k+1}\left(\bar{Y}_{k+1}-\bar{H}_{p p f} \hat{\bar{X}}_{k+1 \mid k}\right) \\
& P_{k+1 \mid k+1}=\left(I-K_{k+1} \bar{H}_{p p f}\right) P_{k+1 \mid k}
\end{aligned}
$$

where, $\hat{\bar{X}}_{k+1 \mid k}, \hat{\bar{X}}_{k+1 \mid k+1}$ are the state prediction and estimate respectively, $P_{k+1 \mid k}, P_{k+1 \mid k+1}$ are the predict covariance and estimate covariance respectively, $K_{k+1}$ is the Kalman gain and

$$
\begin{aligned}
& \bar{H}_{p p f}=\left.\frac{\partial\left(\left[\begin{array}{c}
H\left(x_{k}\right) \\
H\left(x_{k-1}\right) \\
\vdots \\
H\left(x_{k-M+1}\right)
\end{array}\right]\right)}{\partial(\bar{X})}\right|_{\bar{x}=\hat{\bar{X}}_{k+1 \mid k}}=\left[\begin{array}{ccc}
\alpha_{k} & 0 & 0 \\
0 & \ddots & 0 \\
0 & 0 & \alpha_{k-m}
\end{array}\right], \\
& m=0, \cdots, M-1, \\
& \left.\alpha_{k-m}=\left.\left[\begin{array}{cccc}
-\frac{x_{k-m, 1}-x_{k-m}}{r_{k-m, 1}} & -\frac{y_{k-m, 1}-y_{k-m}}{r_{k-m, 1}} & -\frac{z_{k-m, 1}-z_{k-m}}{r_{k-m, 1}} & c \\
-\frac{x_{k-m, 2}-x_{k-m}}{r_{k-m, 2}} & -\frac{y_{k-m, 2}-y_{k-m}}{r_{k-m, 2}} & -\frac{z_{k-m, 2}-z_{k-m}}{r_{k-m, 2}} & c \\
-\frac{x_{k-m, 3}-x_{k-m}}{r_{k-m, 3}} & -\frac{y_{k-m, 3}-y_{k-m}}{r_{k-m, 3}} & -\frac{z_{k-m, 3}-z_{k-m}}{r_{k-m, 3}} & c \\
-\frac{x_{k-m, 4}-x_{k-m}}{r_{k-m, 4}} & -\frac{y_{k-m, 4}-y_{k-m}}{r_{k-m, 4}} & -\frac{z_{k-m, 4}-z_{k-m}}{r_{k-m, 4}} & c
\end{array}\right]\right|_{\bar{x}=\hat{\bar{x}}_{k+11 k}}\right]^{,} \\
& r_{k-m j}=\sqrt{\left(x_{k-m j}-x_{k-m}\right)^{2}+\left(y_{k-m j}-y_{k-m}\right)^{2}+\left(z_{k-m j}-z_{k-m}\right)^{2}} \text {, } \\
& m=0, \cdots, M-1, j=1,2,3,4, \\
& \hat{X}_{k+1}^{1}=\hat{\bar{X}}_{k+1 \mid k+1} \text {, } \\
& P_{k+1}^{1}=P_{k+1 \mid k+1}
\end{aligned}
$$

where 1 denotes local estimate 1 .

Similarly, by applying the extended Kalman filter (EKF) to the existed equation (12) and (17), we may have the expressions of state estimation and covariance for local estimate 2, i.e., $\hat{X}_{k+1}^{2}, P_{k+1}^{2}$

Then we may obtain the global estimation as follows,

$$
\begin{aligned}
& \hat{X}_{k+1}^{g}=\omega_{1} \hat{X}_{k+1}^{1}+\omega_{2} \hat{X}_{k+1}^{2} \\
& \omega_{1}=P_{k+1}^{g} / P_{k+1}^{1} \\
& \omega_{2}=P_{k+1}^{g} / P_{k+1}^{2} \\
& P_{k+1}^{g}=1 /\left(1 / P_{k+1}^{1}+1 / P_{k+1}^{2}\right)
\end{aligned},
$$

\section{SIMULATIONS IN GPS NAVIGATION}

In this section we will give demonstration examples in GPS navigation by both the proposed MSMF and the existed EKF. The software used is Matlab R2010b, which was installed in a notebook PC with OS Windows 7 Professional, Intel i5 CPU $2.50 \mathrm{GHz}$ and 3.00G internal storage.

The process model used in the existed EKF is the wellknown WNA model as follows shown (17) (19)

The satellite data used are as follows [10]

TABLE I GPS SATELLITE DATA

\begin{tabular}{|l|l|l|}
\hline No & $\Omega_{0}$ (deg) & $\theta_{0}$ (deg) \\
\hline 1 & 326 & 68 \\
\hline 2 & 26 & 340 \\
\hline 3 & 146 & 198 \\
\hline 4 & 86 & 271 \\
\hline
\end{tabular}

where, the angle $\Omega_{0}$ is the right ascension of the satellite and $\theta_{0}$ is the angular location of the satellite in its circular orbit. It is assumed that the satellites orbit the earth at a constant rate $\theta$ with a period of approximately 43082s or slightly less than half a day [10]. The equations of motion that describe the angular phasing of the satellites are given, as in the simulation: $\Omega_{k}=\Omega_{0}-\Omega \times t, \theta_{k}=\theta_{0}+\theta \times t$, where, $\Omega=\frac{2 \pi}{86164}, \theta=\frac{2 \pi}{43082}$. In the simulation, the parameter used in the proposed method is $N=1, L=2, M=3$, then according to (9), we obtain $h_{0}=3, h_{1}=-3, h_{2}=1$.

\section{A. Regular situation}

Suppose that the user motion is regular with constant acceleration $a$, initial velocity $V$, and total time simulation is $T$, the time step interval $d T$. In the simulation: $T=150 \mathrm{~s}$, $d T=1 \mathrm{~s}, a=[1 ; 1 ; 1]^{T} \mathrm{~m} / \mathrm{s}^{2}, V=\left[\begin{array}{lll}0 & 0 & 0\end{array}\right] \mathrm{m} / \mathrm{s}$, the process noise covariance in CDF is blkdiag $\left(\sigma_{v 1}^{2}, \sigma_{v 2}^{2}, \sigma_{v 3}^{2}\right)$ with $\sigma_{v 1}^{2}=36, \sigma_{v 2}^{2}=9, \sigma_{v 3}^{2}=1$, the measurement noise covariance is $\sigma_{w}^{2}=36$ for each satellite. Figure 1 shows the absolute estimation error in $x$ position (the $y$ position and $z$ position are similar), where the solid lines with dots represent the results of the existed EKF, the solid lines represent the results of the EKF based on the proposed mode (called PPKF), and the solid lines with "+"s represent the results of the propose MSMF. It can be seen from Figure 1 that all the methods work well. 


\section{B. b. Irregular situation}

Suppose that the acceleration is $a$ within time interval [0, $\left.T_{1}\right]$, and $a_{1}$ within time interval $\left[T_{1}, T\right]$. In the simulation: $T_{1}=60 \mathrm{~s}, \quad a_{1}=10 \times a \quad, \quad a=[3 ; 2 ; 1]^{T} \quad \mathrm{~m} / \mathrm{s}^{2}$, $V=[30 ; 20 ; 10] \mathrm{m} / \mathrm{s}$. The other simulation data are the same as those in regular situation. Figure 2 shows the absolute estimation error in $x$ position (the $y$ position and $z$ position are similar.), where the solid lines with dots represent the results of the existed EKF, the solid lines represent the results of the EKF based on the proposed process mode (called PPKF), and the solid lines with "+"s represent the results of the propose MSMF. It can be seen from Figure 2 that the proposed MSMF and the PPKF still work well when the acceleration is changed, however, the existed EKF does not provide true estimates. Furthermore, the covariance of the MSMF is smaller than that of the PPKF, i.e., 4.739872 vs 5.209839 in simulation.

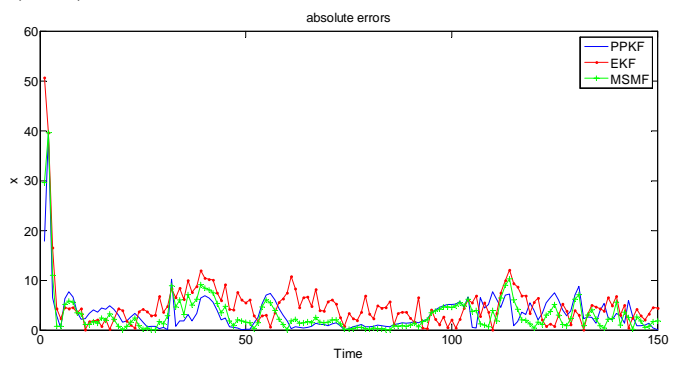

Figure 1. Absolute errors in x position-regular situation

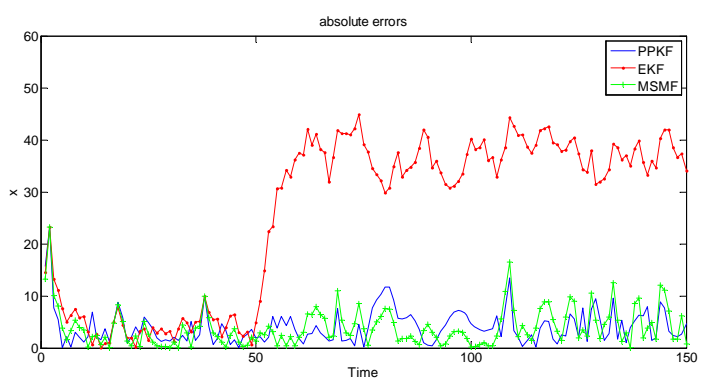

Figure 2. Absolute errors in x position-irregular situation

\section{CONCLUSIONS}

In this paper, firstly we establish a new state space model for GNSS navigation by adopting the idea of state dimension expansion and the PPF, which is an effective way to predict future signal values. And no matter the original state propagation was known or not, we may construct the process model effectively. Then we obtain the two local estimation results by applying the EKF to the original model and the proposed model. Whereafter, according to the weights of covariance, the local results were fused to get the global estimation. Note that the covariance of the global results is smaller than those of local ones. The simulation results demonstrate the good performance of the proposed method over the existed EKF when the state dynamics is not known well.

\section{ACKNOWLEDGMENT}

The work reported in this paper was funded under the foundation of Shanghai Science and Technology Committee (12511500700) and the foundation of the State Key Laboratory of ASIC \& System, Fudan University (11MS006).

\section{REFERENCES}

[1] M. S. Grewal, L. R. Weill, A. P. Andrews, Global positioning systems, inertial navigation, and integration. New York: John Wiley \& Sons, 2001.

[2] S. Yamaguchi, T. Tanaka, GPS standard positioning using Kalman filter. SICE-ICASE International Joint Conference, 2006.

[3] S. S. Hwang, J.L. Speyer, "Particle filters with adaptive resampling technique applied to relative positioning using GPS carrier-phase measurements,” IEEE Transactions on Control Systems Technology, vol. 19, 2011, pp. 1384-1396, doi: 10.1109/TCST.2010.2091415.

[4] H. Guo, M. Yu, C. Zou, W. Huang, "Kalman filtering for GPS/magnetometer integrated navigation system," Advances in Space Research, vol. 45, 2010, pp. 1350-1357.

[5] Y. Koyama, T. K. Liang, T. Tanaka, "High-precision GPS measurement for motorcycle trajectory using Kalman filter," Sixth International Conference on Networked Sensing Systems, 2009.

[6] R. Toledo-Moreo, D. Gruyer, A. Lambert, "A theoretical analysis of the extended Kalman filter for data fusion in vehicular positioning," 11th international conference on ITS telecommunications, 2011.

[7] S. Valiviita, S. J. Ovaska, O. Vainio O, Polynomial predictive filtering in control instrumentation: a review. IEEE Trans. Ind. Electronics, vol. 46, 1999, pp. 876 - 888, doi: 10.1109/41.793335.

[8] J. M. A. Tanskanen, Polynomial predictive filters: implementation and applications. Dissertation, Helsinki University of Technology, 2000.

[9] E. D. Kaplan, C. J. Hegarty, Understanding GPS principles andapplications, Second Edition. Norwood : Artech House, 2006. 